DOI: $10.1002 /$ pamm.202000345

\title{
Evaluation of manufacturing deviations of composite materials
}

\author{
Christian Willberg ${ }^{1, *}$ and Falk Heinecke ${ }^{1}$ \\ ${ }^{1}$ German Aerospace Center, Institute of Composite Structures and Adaptive Systems, Lilienthalplatz 7, \\ 38108 Braunschweig
}

\begin{abstract}
Manufacturing induced imperfections in composites lead to a reduction in the performance of the structure. In order to carry out repairs only when they are really necessary, evaluation capabilities to determine the strength reduction are required. Two methods of evaluation are presented: a finite element based and a Peridynamic based approach, respectively. The finite element based process is used to evaluate stiffness and strength reduction. This processes currently doesn't include progressive failure analysis. Hence, the peridynamics process serves among other things to verify the prediction by the finite element model. Because, the correspondence formulation is used, integral Cauchy stresses are obtained for the laminate and can be verified. Progressive failure analysis is then used to evaluate whether the first ply failure approach used in the finite element model is accurate enough to ensure strength reduction values.
\end{abstract}

() 2021 Wiley-VCH GmbH

\section{Introduction}

The production processes of fibre composites can lead to manufacturing induced deviations. A typical deviation is fibre waviness [4]. Figure 1a shows the failure pattern of a specimen under compressive load with ondulated fibres. Fibre ondulation leads to a local decrease in stiffness and strength. While the change in stiffness is compensated by the surrounding structure the change in strength is an issue. It is known as a nucleus for damage initiation [3]. In a finite element model, see Figure 1b, the samples were modeled to determine effective stiffnesses and strengths [2]. On the one hand, this method is well suited for stiffness determination. On the other hand, the determination of effective strengths require a progressive analysis to account for load redistributions in a multi-layer composite.

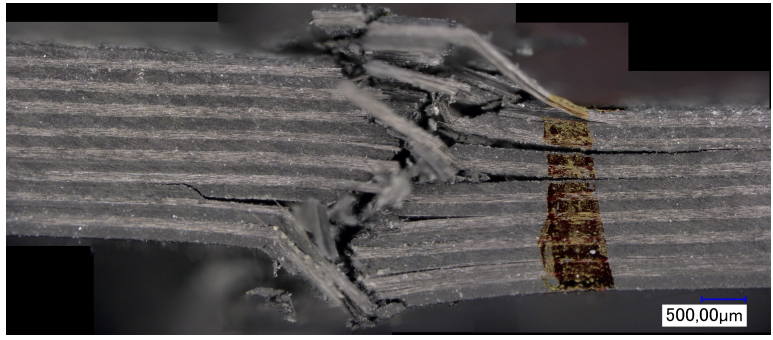

(a) Experimental results.

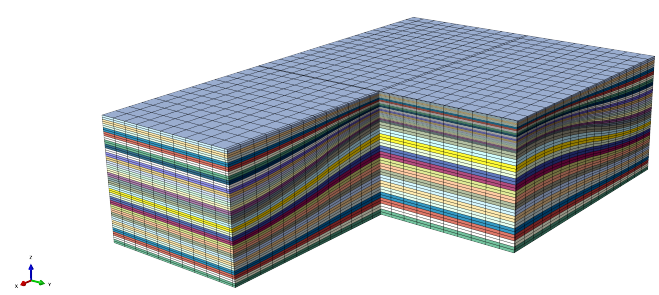

(b) Finite Element model.

Fig. 1: Fibre ondulation.

\section{Approach and Results}

The analysis was performed using Peridynamics. The modelling uses an anisotropic correpondence formulation [1] with a isotropic energy-based damage criterion [6]. The fibre angle $\phi$ distribution is defined as:

$$
\phi=-2 \sin \left(\pi x_{1} / L\right) \cos \left(\pi x_{1} / L\right) \sin ^{2}\left(\pi x_{2} / h\right),
$$

with $L$ as the region of ondulation and $h$ the thickness of the specimen. A special formulation of the non-ordinary state-based model is the so called "correspondence" formulation. It uses an integral deformation gradient:

$$
\boldsymbol{F}=\left[\int_{\mathcal{H}} \underline{\omega}\langle\boldsymbol{\xi}\rangle \underline{\boldsymbol{Y}}\langle\boldsymbol{\xi}\rangle \otimes \underline{\boldsymbol{X}}\langle\boldsymbol{\xi}\rangle d V_{x} \boldsymbol{\xi}\right] \cdot \boldsymbol{K}^{-1} \quad \text { with } \quad \boldsymbol{K}=\int_{\mathcal{H}} \underline{\omega}\langle\boldsymbol{\xi}\rangle \underline{\boldsymbol{X}}\langle\boldsymbol{\xi}\rangle \otimes \underline{\boldsymbol{X}}\langle\boldsymbol{\xi}\rangle d V_{x} \boldsymbol{\xi},
$$

to calculated the bond force density states, where $\boldsymbol{K}$ is the shape tensor, $\underline{X}$ and $\underline{Y}$ are the vector states of the undeformed and deformed bond. With the Cauchy stresses $\sigma$ the force state is then:

$$
\underline{\boldsymbol{T}}\langle\boldsymbol{\xi}\rangle=\underline{\omega}\langle\boldsymbol{\xi}\rangle \boldsymbol{\sigma} \boldsymbol{K}^{-1} \boldsymbol{\xi} .
$$

* Corresponding author: e-mail christian.willberg@ dlr.de, phone +49531295 2489, fax +495312952232 
In the correspondence models so called zero-energy modes could occur, which are a result of the integral determination of the deformation gradient. This zero-energy modes lead to unstable solutions. Several stabilization methods were published to overcome this problem. Wan et al. [5] propose a promising approach. The corrected force state $\underline{T}^{C}$ with suppression of the zero-energy mode is:

$$
\underline{\boldsymbol{T}}^{C}=\underline{\boldsymbol{T}}+\underline{\boldsymbol{T}}^{S}
$$

where $\underline{T}$ is given in Equation 3. According to Wan et al. [5] the suppression force density state $\underline{T}^{S}$ can be formulated as:

$$
\underline{\boldsymbol{T}}^{S}\langle\boldsymbol{\xi}\rangle=\underline{\omega}\langle\boldsymbol{\xi}\rangle \boldsymbol{C}_{1}(\underline{\boldsymbol{Y}}\langle\boldsymbol{\xi}\rangle-\boldsymbol{F} \boldsymbol{\xi}) \quad \text { with } \quad \boldsymbol{C}_{1}=\boldsymbol{C} \cdots \boldsymbol{K}^{-1} .
$$

$\underline{\boldsymbol{Y}}\langle\boldsymbol{\xi}\rangle-\boldsymbol{F} \boldsymbol{\xi}$ caused by the zero-energy mode. If the approximated deformation gradient $\boldsymbol{F}$ exact no extra deformation and therefore no zero-energy mode occur. Figure 2 a shows exemplarily the failure pattern and damage index of a specimen with ondulated fibres. Figure $2 b$ provides force-deformation curves for ondulations with different orders of magnitude. The results at the undirectional specimen show that the stiffness decrease is as expected with increasing out-of-plane fibre angle. At the same time, damage initiation takes place earlier due to pressure-bending superposition.

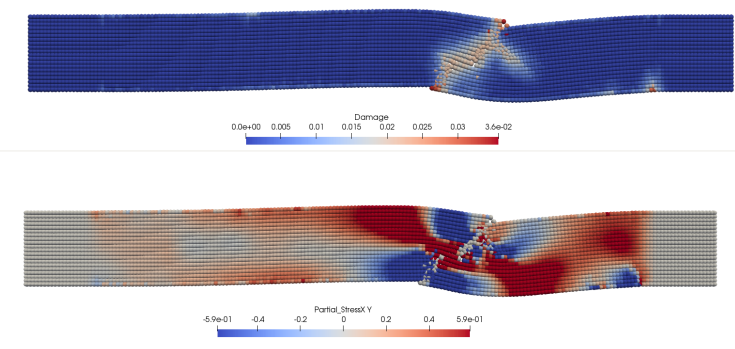

(a) Shear stresses and damage indices of a $10^{\mathrm{deg}}$ model.

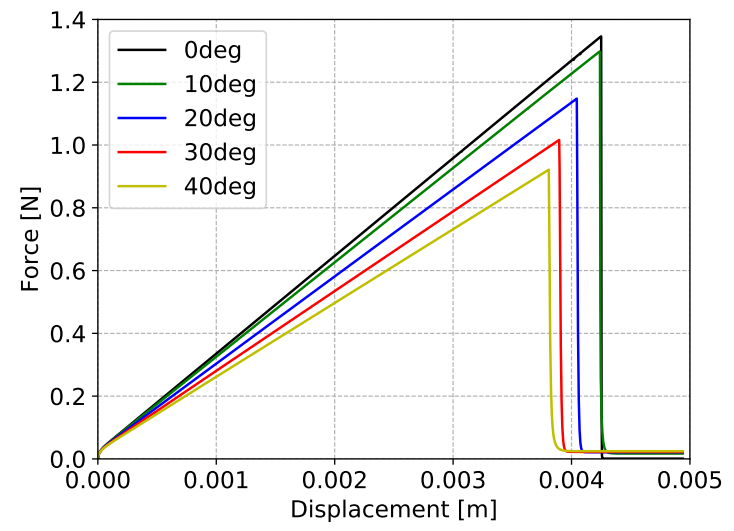

(b) Force displacement curve for different angles.

Fig. 2: Numerical results.

\section{Conclusion}

In Abstract it could be shown that the damage behaviour of unidirectional wavy specimens could be qualitatively realized. The way forward is to adapt the models to the measured data and to analyze quasi-isotropic laminates.

\section{References}

[1] F. Bobaru, J. T. Foster, P. H. Geubelle, and S. A. Silling, Handbook of peridynamic Modeling (CRC Press, 2016), p. 530.

[2] F. Heinecke, and T. Wille, CEAS Aeronautical Journal 9, 123-133 (2018)

[3] W. Woigk, S. R. Hallett, M. I. Jones, M. Kuhtz, A. Hornig, and M. Gude, Journal of Composite Structures 201, 1004-1017 (2018).

[4] F. Heinecke, and C. Willberg, Journal of Composite Science 2, 56 (2019)

[5] J. Wan, Z. Chen, X. Chu, and H. Liu, Acta Mechanica Sinica 35, 1021-1032 (2019)

[6] C. Willberg, M. Rädel, and L. Wiedemann, Journal of Mechanics of Materials and Structures 14, 193-217 (2019) 